\title{
Aminoglycoside antibiotics restore dystrophin function to skeletal muscles of mdx mice
}

\author{
Elisabeth R. Barton-Davis, ${ }^{1}$ Laurence Cordier, ${ }^{1}$ Daria I. Shoturma, ${ }^{1}$ \\ Stuart E. Leland, ${ }^{2}$ and H. Lee Sweeney ${ }^{1}$ \\ ${ }^{1}$ Department of Physiology, and \\ ${ }^{2}$ Institute for Human Gene Therapy, University of Pennsylvania School of Medicine, Philadelphia, Pennsylvania 19104, USA
}

Address correspondence to: H. Lee Sweeney, A700 Richards Building, Department of Physiology, 3700 Hamilton Walk, University of Pennsylvania School of Medicine, Philadelphia, Pennsylvania 19104-6085, USA. Phone: (215) 898-0486;

Fax: (215) 898-0475; E-mail: Lsweeney@mail.med.upenn.edu.

Received for publication July 14, 1999, and accepted July 16, 1999.

Duchenne muscular dystrophy (DMD) is caused by mutations in the dystrophin gene, leading to the absence of the dystrophin protein in striated muscle. A significant number of these mutations are premature stop codons. On the basis of the observation that aminoglycoside treatment can suppress stop codons in cultured cells, we tested the effect of gentamicin on cultured muscle cells from the mdx mouse - an animal model for DMD that possesses a premature stop codon in the dystrophin gene. Exposure of $m d x$ myotubes to gentamicin led to the expression and localization of dystrophin to the cell membrane. We then evaluated the effects of differing dosages of gentamicin on expression and functional protection of the muscles of $m d x$ mice. We identified a treatment regimen that resulted in the presence of dystrophin in the cell membrane in all striated muscles examined and that provided functional protection against muscular injury. To our knowledge, our results are the first to demonstrate that aminoglycosides can suppress stop codons not only in vitro but also in vivo. Furthermore, these results raise the possibility of a novel treatment regimen for muscular dystrophy and other diseases caused by premature stop codon mutations. This treatment could prove effective in up to $15 \%$ of patients with DMD.

J. Clin. Invest. 104:375-381 (1999).

\section{Introduction}

Duchenne muscular dystrophy (DMD) is an X-linked degenerative disorder of muscle caused by the absence of the protein dystrophin. The disease affects approximately 1 in 3,500 male newborns. Approximately one third of the cases arise from new mutations in the dystrophin gene (1). This is due, in part, to the large size of the dystrophin gene (2.4 megabases; reference 2$)$, which makes it susceptible to spontaneous mutations. Roughly half of these mutations have been shown to be deletions within the gene (3). However, $60 \%$ of the remaining cases are thought to be point mutations, which include mutations that lead either to splicing errors or to premature stops in the polypeptide coding sequence (4). A study of 158 patients with nondeletion mutations found that 16 of the patients had a premature stop codon in the dystrophin coding sequence (5). The $m d x$ mouse, which serves as an animal model for DMD, contains a point mutation (6). This mutation causes premature termination of the protein and leads to an absence of dystrophin and the dystrophin-associated protein complex within the muscle cell membrane. Muscles from the $m d x$ mouse exhibit critical hallmarks of the human form of the disease, including a high susceptibility to contractile-induced damage, a significant degree of muscle fiber degeneration and regeneration, and increased fibrosis later in life (7-9). The $m d x$ mouse thus serves as both a phenotypic and genotypic model for those patients in whom DMD arises from premature stop codons. The suppression of premature stop codons can be mediated by aminoglycosides in cultured cells $(10,11)$. These antibiotics cause extensive misreading of the RNA code and can thereby allow the insertion of alternative amino acids at the site of the mutated codon (10). Recent reports have shown that when cultured cells exhibiting premature stop mutations in the cystic fibrosis transmembrane conductance regulator (CFTR) are treated with aminoglycosides, synthesis of the full-length protein is restored $(12,13)$. Although this has never been demonstrated in vivo, it raises the possibility that any disease caused by point mutations might be treated using aminoglycosides. We tested this hypothesis in primary muscle cultures from the $m d x$ mouse, as well as on the whole animal. We have found that gentamicin treatment results in the synthesis and proper localization of dystrophin both in vitro and in vivo. More importantly, the functional protection of dystrophin against contraction-induced damage is observed in gentamicin-treated muscles of $m d x$ mice. Therefore, aminoglycosides could represent a new avenue of treatment in muscular dystrophy and other diseases caused by premature stop codon mutations.

\section{Methods}

Gentamicin treatment in primary cell culture. Mouse primary skeletal muscle cultures were derived from the muscles of 1-day-old $m d x$ mice as described previously (14). Cells were cultured for 10 days in the presence of $0-500$ $\mu \mathrm{g} / \mathrm{mL}$ gentamicin sulfate (GIBCO BRL, Grand Island, 
New York, USA). Culture medium was replaced every 4 days. The presence of dystrophin in myoblast cultures was detected by immunostaining as described previously (15). A primary mAb to dystrophin (F192A12; Alexis Corp., San Diego, California, USA) was used undiluted. Rhodamine-conjugated anti-mouse IgG (Jackson ImmunoResearch Laboratories Inc., West Grove, Pennsylvania, USA) was used as the secondary antibody. Staining was viewed using a Leica DMR microscope (Leica Microsystems Inc., Deerfield, Illinois, USA), digital camera, and associated imaging software (OpenLab, Improvision, Coventry, United Kingdom).

Gentamicin treatment in mice. Using pharmacokinetic data generated in the dog, the allometric dose equivalent (16) for gentamicin sulfate (Boehringer Ingelheim, Ridgefield, Connecticut, USA) in mice was calculated at 17 $\mathrm{mg} / \mathrm{kg}$. Male $m d x$ mice were given a subcutaneous injection of $100 \%, 200 \%$, and $400 \%$ of the calculated dose equivalent once per day for 14 days. Control groups included uninjected male $m d x$ and male C57BL/6J mice and male C57BL/6J mice injected with $200 \%$ of the calculated dose equivalent for gentamicin sulfate.

In a separate experiment, gentamicin treatment was administered by Alzet osmotic pumps (Alza Corp., Palo Alto, California, USA). Osmotic pumps were implanted under the skin of anesthetized mice per the manufacturer's instructions. The pumps were loaded with appropriate drug concentrations for the mice to receive 50\%, $100 \%$, and $200 \%$ of the calculated dose for 2 weeks.

At the end of the treatment regimen, animals were anesthetized with ketamine (Phoenix Pharmaceuticals Inc., Mountain View, California, USA) and exsanguinated. The kidney, heart, diaphragm, tibialis anterior (TA) muscles, and extensor digitorum longus (EDL) muscles were removed for subsequent analysis. Serum was analyzed for creatine kinase (CK) activity (Sigma Chemical Co., St. Louis, Missouri, USA) and also for peak and trough gentamicin levels using a fluorescence polarization assay (Diagnostic Laboratory, Cornell University, New York, New York, USA). Kidneys were examined for evidence of renal nephrosis as an assay of aminoglycoside toxicity (M.S. Hershey Medical Center, Hershey, Pennsylvania, USA) (17). Heart, diaphragm, and TA were rapidly frozen in melting isopentane for immunofluorescence analysis of dystrophin incorporation into striated muscles. EDL muscles were analyzed for functional performance as described below.

Muscle mechanics measurements. Isolated whole-muscle mechanics were performed on EDL muscles from drugtreated and control animals using a previously described apparatus (18). Protection against mechanical injury, induced by a series of eccentric contractions with stretches of $10 \%$ of optimal length $\left(\mathrm{L}_{\mathrm{o}}\right)(8)$, was evaluated. Damage was determined as the loss in force between the first and last eccentric contraction. At the end of the mechanical measurements, muscles were blotted, weighed, and then rapidly frozen in melting isopentane for subsequent analysis. During the mechanical injury protocol, Procion orange (a low-molecular-weight, membraneimpermeant dye) was present in the medium bathing the muscles. Thus, further confirmation of muscle damage was obtained by measuring the entry of Procion orange into damaged EDL fibers, as described previously (8).

Immunohistochemistry. The presence and membrane localization of both dystrophin and $\gamma$-sarcoglycan (a member of the dystrophin glycoprotein complex; reference 19) were observed by immunofluorescence on $10-\mu \mathrm{m}$ frozen sections of striated muscles. The primary antibody for dystrophin $(60 \mathrm{kDa}$; a kind gift from Eric Hoffman, University of Pittsburgh, Pittsburgh, Pennsylvania, USA; reference 20 ) was diluted $1: 500$ in $10 \%$ horse serum, and the secondary antibody (FITC-conjugated donkey anti-sheep; Jackson ImmunoResearch Laboratories Inc.) was diluted 1:200. A $\gamma$-sarcoglycan polyclonal antibody (kind gift of Elizabeth McNally, University of Chicago, Chicago, Illinois, USA; reference 21) was diluted $1: 600$ in $5 \%$ BSA, and the secondary antibody (FITC-conjugated donkey anti-rabbit; Jackson ImmunoResearch Laboratories Inc.) was diluted 1:3,000. Stained sections were viewed on a Leica DMR microscope and captured with a digital camera using image analysis software (OpenLab).

Immunoblotting. An estimate of restoration of the dystrophin glycoprotein complex to the sarcolemma was performed by immunoblotting of muscle homogenates. Antibodies to both dystrophin (COOH-terminal antibody; Dys2 [Novacastra Laboratories Ltd., Newcastle upon Tyne, United Kingdom]) and $\gamma$-sarcoglycan were used for this measurement.

\section{Results}

To determine whether gentamicin could lead to suppression of the premature stop codon in dystrophin in the $m d x$ mouse, primary myotubes were cultured from neonatal $m d x$ mice in the presence of gentamicin. Dystrophin was detected in 14-day cultures treated with $300 \mu \mathrm{g} / \mathrm{mL}$ gentamicin (Figure 1 and Table 1 ). The myotubes displayed definitive sarcomeres, indicative of a differentiated state. Moreover, spontaneous contractions were observed at this treatment level. Lower doses did not result in detectable dystrophin protein. At higher levels of gentamicin treatment, myotube size

Table 1

Gentamicin treatment promotes dystrophin expression in $m d x$ myotubes

\begin{tabular}{|c|c|c|c|c|c|c|}
\hline $\begin{array}{l}\text { Gentamicin } \\
(\mu \mathrm{g} / \mathrm{mL})\end{array}$ & $\begin{array}{c}\text { Control } \\
(0)\end{array}$ & $\begin{array}{c}100 \\
(\mu \mathrm{g} / \mathrm{mL})\end{array}$ & $\begin{array}{c}200 \\
(\mu \mathrm{g} / \mathrm{mL})\end{array}$ & $\begin{array}{c}300 \\
(\mu \mathrm{g} / \mathrm{mL})\end{array}$ & $\begin{array}{c}400 \\
(\mu \mathrm{g} / \mathrm{mL})\end{array}$ & $\begin{array}{c}500 \\
(\mu \mathrm{g} / \mathrm{mL})\end{array}$ \\
\hline Presence of myotubes & +++ & +++ & +++ & ++ & + & - \\
\hline Myotube size & +++ & +++ & +++ & ++ & + & - \\
\hline Dystrophin staining & - & - & - & + & + & - \\
\hline
\end{tabular}

Values are relative to levels of $C 57$ myotubes, which are $(+++)$ for all parameters in the absence of gentamicin. 


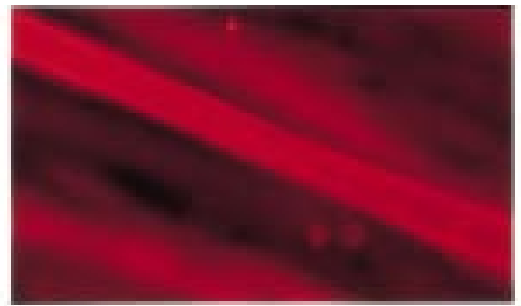

C57

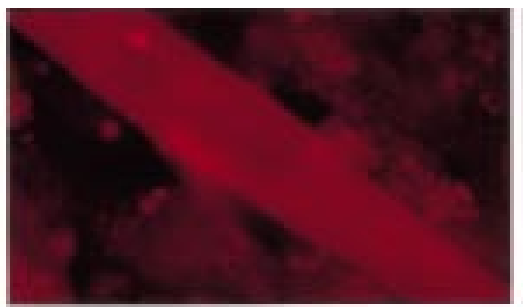

Treated mdx

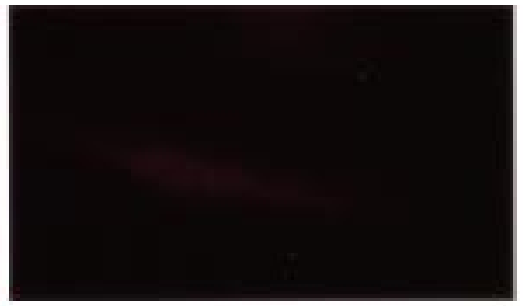

Control mdx

Figure 1

Immunohistochemistry of myotubes from primary cell culture from C57 and $m d x$ muscle. The presence of dystrophin was detected by mAb to the $\mathrm{COOH}$-terminus of dystrophin (F192A12) followed by a rhodamine-conjugated anti-mouse IgG. Dystrophin was present in myotubes from C57 mice (left) and in $m d x$ myotubes treated with $300 \mu \mathrm{g} / \mathrm{mL}$ (middle). No dystrophin was detected in untreated mdx myotubes (right).

and number diminished to a point at which no myotubes developed $(500 \mu \mathrm{g} / \mathrm{mL})$. This suggests that there is a window of gentamicin dosage in which misreading can lead to the restoration of full-length dystrophin synthesis. However, excessive levels of drug can interfere with translation to the point that no proliferation and differentiation can occur.

\section{Figure 2}

The effect of gentamicin treatment on eccentric contraction injury. Effective treatment regimes are depicted in $\mathbf{a}$. The decrement in force after 5 eccentric contractions was significantly less in $m d x$ EDL muscles injected with gentamicin (mdxGi200, $n=8 ; 200 \%$ dose equivalent) compared with untreated $m d x$ EDLs (mdxctrl, $n=8$ ) and muscles from Becker construct (22) transgenic animals (Beck tg, $n=5$ ). The level of protection afforded by gentamicin in $m d x$ muscles approached the values measured in EDLs from untreated C57 mice ( $C 57 \mathrm{ctrl}, n=4)$, and was not significantly different from EDLs from gentamicin-treated C57 mice (C57Gi200, $n=4)$. DHB treatment, when combined with 200\% gentamicin (mdxGi200D, $n$ $=4$ ), did not alter levels of protection by this gentamicin dose alone. Lower injected doses of gentamicin (50\% dose equivalent) were very effective at preventing force decrements in female $m d x$ mice ( $\mathrm{mdxGi50F}, n=$ 4). Treatment regimens that were not effective in preventing eccentric contractile injury in male $m d x$ mice are displayed in b. These include $400 \%$ gentamicin injected in combination with DHB ( $m d x G i 400 \mathrm{D}, n=4), 100 \%$ injected gentamicin ( $m d x G i 100, n=4$ ), and all $m d x$ animals treated by infusion pumps (mdxGP50, 50\% dose; mdxGP100, 100\% dose; mdxGP200, 200\% dose; $n=4$ for each condition). The measurements of force generation after eccentric contraction were reflected in the measurements of membrane damage $(\mathbf{c})$. There was a significant reduction in the proportion of fibers with dye entry in EDLs from treated $m d x$ mice compared with muscles from untreated $m d x$ muscles. ${ }^{\dagger} P<0.05$ for comparisons between treated $m d x$ muscles and untreated C57 controls. ${ }^{*} P<$ 0.05 for comparisons between treated and untreated $m d x$ muscles.
Next, we examined a range of gentamicin doses in male and female $m d x$ mice. We first looked for functional evidence of dystrophin protection against contraction-induced damage. This involved an eccentric contraction protocol that was used to evaluate muscles from gentamicin-treated and control animals, including control $m d x$ mice expressing a truncated dys-

\section{a}

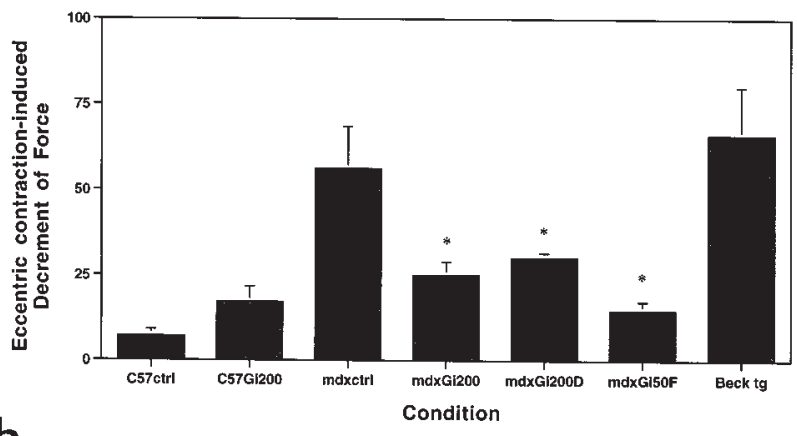

b

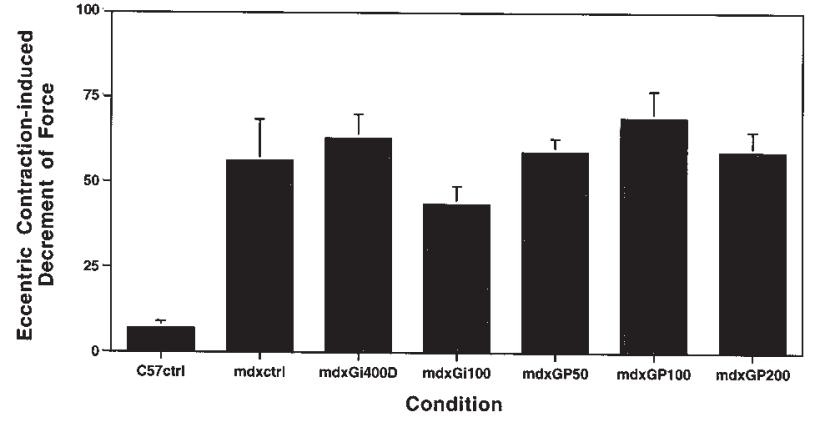

c

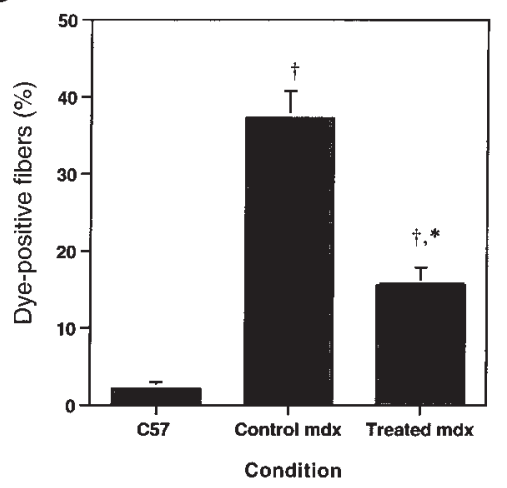



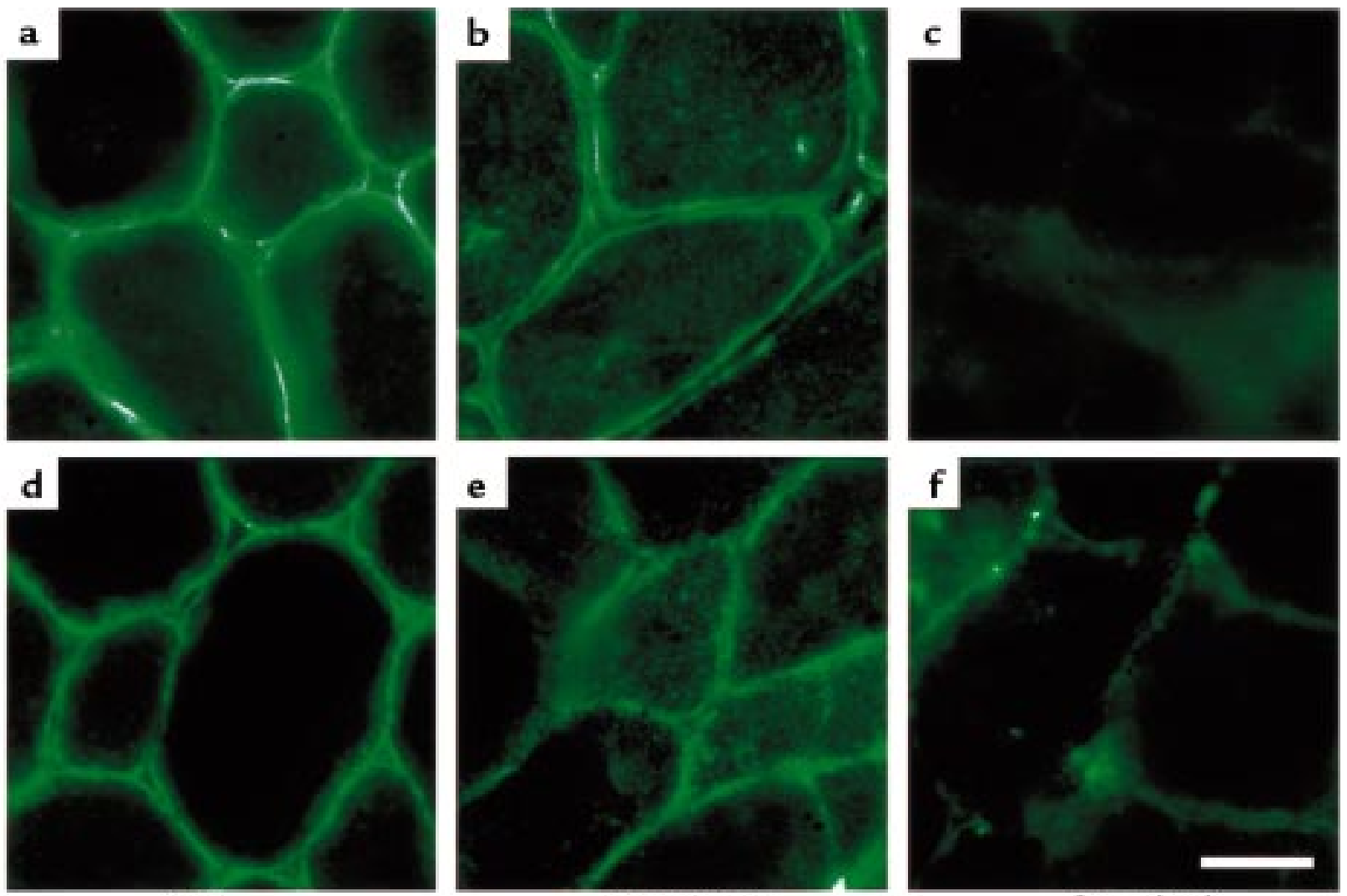

Treated mdx

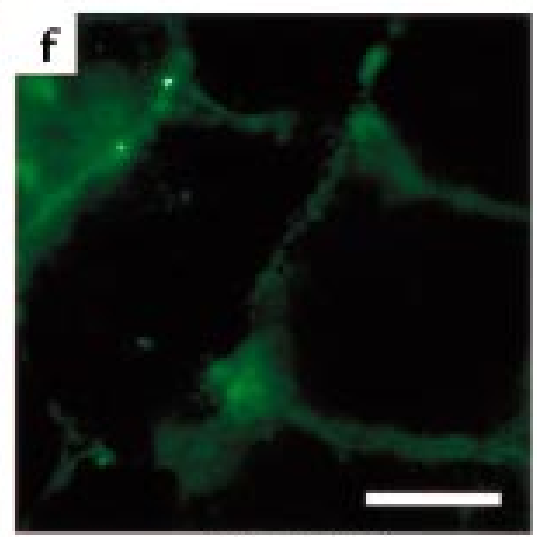

Control mdx

Figure 3

Immunohistochemistry of muscle cross-sections to view dystrophin (a-c) and $\gamma$-sarcoglycan (d-f). C57 control TA displayed positive staining for both proteins ( $\mathbf{a}$ and $\mathbf{d}$ ). Lower staining intensity for dystrophin and $\gamma$-sarcoglycan was observed in $m d x$ TAs treated with gentamicin (b and $\mathbf{e})$. No dystrophin staining, and only minimal staining of $\gamma$-sarcoglycan, were seen in $m d x$ control TA muscles (c and $\mathbf{f}$ ). Scale bar: $25 \mu \mathrm{m}$.

trophin (22). The results of these measurements are summarized in Figure 2, a and b. Significant protection against contractile injury was observed in 7 of 8 EDLs from male $m d x$ mice treated at the $200 \%$ dose equivalent. In female $m d x$ mice, comparable protection was achieved at the 50\% dose equivalent (Figure 2a). Gentamicin treatment at other injected doses, or continuous release of drug by osmotic pumps, did not afford any significant protection against eccentric contraction-induced injury (Figure $2 \mathrm{~b}$ ).

Functional protection against injury was also confirmed by viewing of sarcolemmal damage to the muscles, as evaluated by Procion orange entry during the contraction-induced damage protocol. This fluorescent dye can enter muscle cells only through membrane disruptions, and it therefore serves as an indicator of transient breaks in the sarcolemma (8). The decreases in force generation during eccentric contractions correlated with the amount of sarcolemmal damage found in all muscles, regardless of treatment group. This result is shown in Figure 2c for muscle cross-sections from C57, control $m d x$, and treated (200\% dose equivalent) male $m d x$ EDLs that had been subjected to eccentric contractions in the presence of Procion orange. The number of damaged fibers in treated $m d x$ muscles were signifi- cantly fewer than in control $m d x$ muscles. The results from functional and structural analysis support the hypothesis that gentamicin treatment can protect $m d x$ muscle from contraction-induced injury.

We next confirmed that the mechanism by which gentamicin leads to protection against injury is through the restoration of dystrophin. To address whether gentamicin treatment enabled translation and stabilization of full-length dystrophin, immunohistochemistry was performed on muscle cross-sections. In all cases, EDL muscles that demonstrated protection against contraction-induced injury also displayed appreciable staining of dystrophin compared with EDL muscles from untreated $m d x$ mice and compared with those treated muscles in which there was no protection against injury. Furthermore, in the animals that demonstrated functional protection, we saw membrane localization of dystrophin in all muscles examined, including TA, heart, and diaphragm. Cross-sections of TA muscles are shown in Figure 3.

To test whether the assembly of the dystrophin-associated glycoprotein complex (DGC) was restored by gentamicin treatment, immunohistochemistry of $\gamma$-sarcoglycan (19) was performed. The presence of $\gamma$-sarcoglycan was detected in all muscles that were positive for dys- 


\section{Figure 4}

Immunoblot analysis of TA muscles for dystrophin and $\gamma$-sarcoglycan. Gentamicin treatment at the $200 \%$ dose equivalent significantly increased both dystrophin and $\gamma$-sarcoglycan levels above those in untreated $m d x$ muscle (lane 2) when introduced by injection (lane 3 ) or by infusion pump (lane 5) into male $m d x$ mice. The protein levels were approximately $10-20 \%$ those of C 57 controls (lane 1 ). Female $m d x$ mice treated with only $50 \%$ doses of gentamicin (lane 4 ) also displayed significant levels of both proteins compared with untreated controls.

trophin, including those of the heart, diaphragm, and TA - indicative of the restoration of the DGC. (TA staining is depicted in Figure 3.) However, as is evident in Figure 3 , the gentamicin-treated animals show some cytoplasmic staining for both dystrophin and $\gamma$-sarcoglycan. Thus, not all of the dystrophin complexes are membrane localized and likely to be functional.

Western blots (Figure 4) of either dystrophin (using a $\mathrm{COOH}$-terminal antibody to detect full-length protein) or $\gamma$-sarcoglycan revealed that the level in the treated male $m d x$ TA muscles given the $200 \%$ dose equivalent, or in females given the $50 \%$ dose equivalent, was $10-20 \%$ of the level in C57 muscles. The return of dystrophin and its associated complex was observed in all striated muscles that we examined, including TA, EDL, diaphragm, and the heart. Thus, systemic delivery of gentamicin can promote the synthesis of functional dystrophin in all striated muscles.

Paradoxically, the highest levels of dystrophin in the gentamicin-treated $m d x$ mice were in the group of male mice given the $200 \%$ dose equivalent by infusion pump. Although these muscles were highly susceptible to contraction-induced injury (Figure $2 \mathrm{~b}$ ), their muscles contained approximately $20 \%$ of normal dystrophin levels.

To obtain a further indication of the degree of protection resulting from gentamicin treatment, we examined serum CK levels on a subset of the mice. As shown in Figure 5, the blood CK levels for the gentamicin-treated male mice (200\% dose equivalent) were not significantly elevated compared with control (C57) mice. In the $m d x$

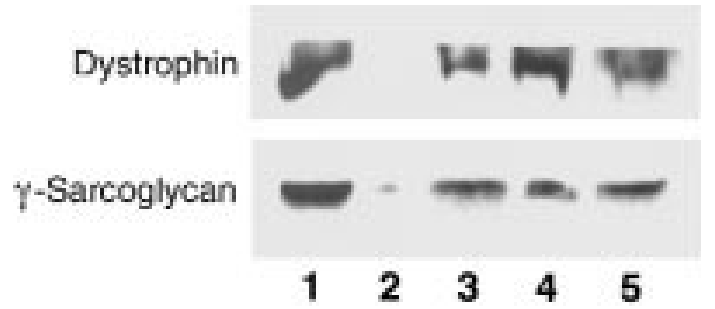

mice, the CK levels were substantially elevated (Figure 5). Thus, the level of protection afforded by the drug treatment was sufficient to return CK levels to normal.

Although aminoglycosides are used routinely in the treatment of bacterial infections, there are possible side effects that need to be monitored. These include nephrotoxicity and ototoxicity (17). Previous studies have shown that iron chelators, such as 2,3-dihydroxybenzoate (DHB), used in combination with gentamicin prevent hearing loss associated with aminoglycoside use (23), and do not interfere with the use of gentamicin as an antibiotic (24). Accordingly, we examined the combined use of DHB and gentamicin (males with 200\% dose equivalent). This resulted in functional protection (force decrements were attenuated) in 4 of 6 animals (Figure 2a). The muscles from the remaining 2 animals showed no protection against eccentric contraction. Muscles from male $m d x$ mice receiving DHB plus $400 \%$ gentamicin dose equivalent were afforded no protection against eccentric contraction, consistent with results from administration of gentamicin alone. These results suggest that DHB does not interfere with the ability of gentamicin to restore dystrophin function, and that it may even prevent toxic side effects.

To address the potential renal complications associated with daily injections of gentamicin, mice were examined for signs consistent with uremia, such as depression, decreased activity levels, and decreased appetite. Mice appeared normal throughout the treatment regimen. At the end of the injection series, kidneys were

\section{Figure 5}

The effect of gentamicin treatment on serum CK levels. The levels of serum CK in untreated $m d x$ mice $(n=4)$ were significantly reduced in $m d x$ mice treated with $200 \%$ gentamicin by daily injection $(n=4)$. These levels approached those measured in C57 control mice $(n=2)$. ${ }^{+} P<0.05$ for comparisons between treated $m d x$ muscles and untreated C57 controls. ${ }^{*} P<0.05$ for comparisons between treated and untreated $m d x$ muscles.

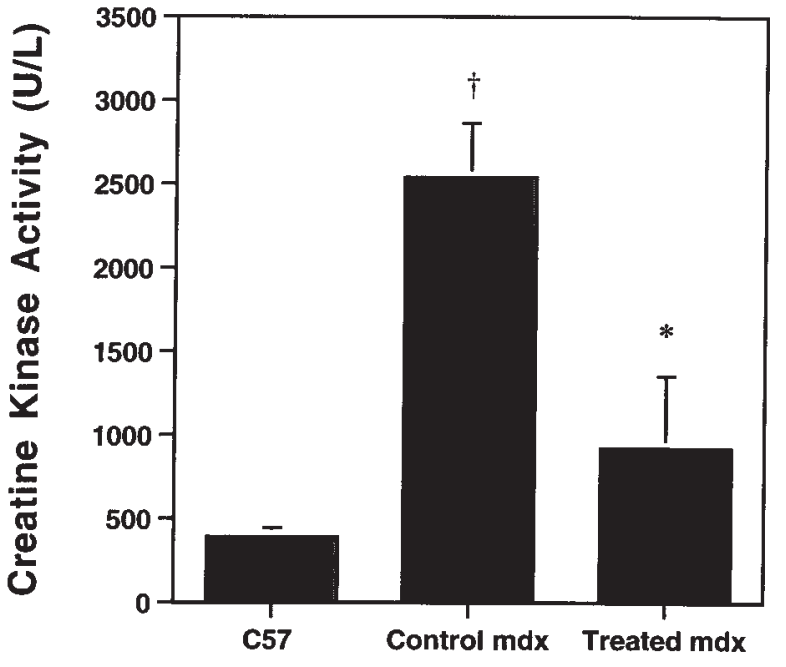


examined histologically, and no pathology was observed in the kidneys from any treatment group. Peak serum gentamicin levels $(65.0 \pm 5.8 \mu \mathrm{g} / \mathrm{mL} ; n=3)$ were observed 20 minutes after injection and decreased rapidly to below $1 \mu \mathrm{g} / \mathrm{mL}$ by $4-24$ hours after injection. (Toxicity and clearance impairment levels are approximately 10 $\mu \mathrm{g} / \mathrm{mL}$ in humans.) Serum gentamicin levels from male $m d x$ mice given the drug by infusion pump were $2.5 \pm 0.2$ $\mu \mathrm{g} / \mathrm{mL}(n=4)$ at the time of analysis.

\section{Discussion}

To our knowledge, these experiments are the first to provide in vivo evidence of phenotypic suppression of a premature stop codon by aminoglycosides. These results hold great promise for the treatment of a subset of the DMD population, as well as other diseases caused by such nonsense mutations.

Although gene therapy is seen as the future hope for the treatment of DMD and other genetic diseases (25, 26), aminoglycoside antibiotics offer a different and immediate approach to the treatment of genetic disease. Gentamicin injections provide systemic delivery of the therapeutic agent, which, as the results described herein demonstrate, can achieve phenotypic suppression throughout the animal. This is a distinct advantage over the presently more directed approach taken by gene therapy, particularly in muscular dystrophy, in which the defect is in all muscles of the body. Although initial studies of adenoviral gene transfer of dystrophin show that the restoration of subnormal dystrophin levels in approximately $50 \%$ of the fibers can protect striated muscle from damage (27), it is clear that delivery of the gene transfer vehicle is a major unresolved problem. On the other hand, systemic delivery of aminoglycoside is simple and can potentially restore dystrophin to all fibers in all muscles.

Aminoglycosides can restore only a fraction of normal levels of full-length protein, as shown in Figure 4. Studies of transgenic $m d x$ mice expressing full-length dystrophin over a range of normal levels have demonstrated that the restoration of $20 \%$ normal dystrophin levels can prevent symptoms of the disease (28). In the same study, mice with significantly lower levels $(<5 \%)$ of full-length dystrophin still displayed a reduction of symptoms. We estimate that dystrophin levels in gentamicin-treated $m d x$ muscle are approximately $10-20 \%$ of the levels in normal muscle. This suggests that mistranslation cannot completely restore normal levels of protein. Although there is a significant degree of functional protection against injury observed in these muscles, compared with untreated $m d x$ muscles (Figure 2), complete functional restoration was not achieved. Our experiments are consistent with the transgenic studies: although low levels of dystrophin expression can afford significant protection to diseased muscle, there is a critical level of expression that must be reached in order to restore full function. To put this in perspective, however, the level of protection achieved with gentamicin is much greater than the protection afforded by overexpression of a truncated dystrophin derived from a patient with mild Becker muscular dystrophy. The same mechanical damage protocol applied to transgenic mice expressing this truncated dystrophin (22) results in a level of damage that is indistinguishable from that seen in $m d x$ muscles (Figure $2 \mathrm{a}$ ).

The fact that some animals do not respond to the gentamicin treatment suggests that there is a critical level of gentamicin required to obtain significant translation of dystrophin. Either mouse-to-mouse variability in metabolizing gentamicin, or variability in injection, could account for the small number of animals that did not benefit from treatment. The results of injections into female $m d x$ mice suggest that the rate of drug metabolism is a likely source of variability. In female $m d x$ mice, the dose equivalent that provided dystrophin restoration was $50 \%$, rather than $200 \%$. This is consistent with the lower rate of metabolism of drugs in females.

Thus, it is clear from both our in vitro and in vivo experiments that there is a narrow window in which gentamicin treatment is effective in restoring dystrophin function to $m d x$ muscle. Intuitively, one can imagine that below a certain level, mistranslation will not occur, and that above some level, the rate of misreads will be too high to allow functional protein synthesis. However, the goal would be to achieve a level that promotes infrequent misreads, and perhaps preferential misreads, at a premature stop codon where there is not a competition with a "correct" tRNA.

While we identified a daily dose equivalent that seems to have realized this goal in mice, the frequency of drug delivery was also critical. It appears that a daily bolus injection may be optimal. Certainly it seems preferable to sustained release. When we delivered the same dose equivalent that was effective in 1 bolus dose per day via sustained release with osmotic pumps, it resulted in no functional protection (Figure $2 \mathrm{~b}$ ), even though considerable amounts of dystrophin were produced (Figure 4). This experiment provides insight into a key feature of gentamicin treatment. We hypothesize that the "spike" of gentamicin in the blood immediately after injection allows significant amounts of mistranslation and, thus, the ability to suppress a stop codon. On the other hand, a rapid return to low blood levels of drug may be necessary for preventing widespread mistranslation of proteins and, thus, undesirable side effects. In the mice injected with the $200 \%$ dose, serum levels were at a maximum $(65.0 \mu \mathrm{g} / \mathrm{mL})$ for less than 2 hours. The drug was rapidly metabolized and approached minimum values $(<1 \mu \mathrm{g} / \mathrm{mL})$ by 4 hours after injection. In mice, we have shown that the spike must reach at least $40 \mu \mathrm{g} / \mathrm{mL}$ for the duration of the peak phase, as lower injected doses did not increase dystrophin.

It is unclear what serum level must be achieved in humans for gentamicin to suppress stop codons effectively. However, trials with escalating dosages given once a day can be coupled with daily monitoring of serum CK levels. Based on our mouse studies, it is possible that daily administration of gentamicin at levels that are below the maximum recommended human dosage could prove effective in restoring dystrophin function. Obviously, such trials only can involve patients with confirmed premature stop codons, as no other patients with DMD will derive benefit.

Earlier studies of phenotypic suppression by amino- 
glycosides explored a number of these antibiotics, as well as specific forms of each drug with various side-chain substitutions (11). Active forms of gentamicin include $\mathrm{X}_{2}, \mathrm{C}_{1 \mathrm{a}}, \mathrm{C}_{2}$, and $\mathrm{A}$. Standard pharmaceutical mixtures of gentamicin contain approximately $50 \%$ of the isoforms that are active in phenotypic suppression. It is possible that tailoring aminoglycoside composition to suit the task of phenotypic suppression might increase the efficiency of this avenue of treatment. For gentamicin to become part of a standard treatment for diseases with premature stop codons, this and a number of other issues must be addressed, including long-term use, different treatment regimens, and toxicity. However, given that there is no treatment for DMD and that the use of gentamicin in humans is already well established, this new and potentially very exciting treatment should be readily tested in appropriate patients. This may be up to $15 \%$ of all DMD patients (29).

\section{Acknowledgments}

This work was funded by grants from the National Institutes of Health (AR/NS43648) and the Muscular Dystrophy Association. E.R. Barton-Davis and L. Cordier are funded by fellowships from the Muscular Dystrophy Association. H.L. Sweeney acknowledges valuable discussions with J. Woo and A. Stewart.

1. Moser, H. 1984. Duchenne muscular dystrophy: pathogenic aspects and genetic prevention. Hum. Genet. 66:17-40.

2. Koenig, M., Monaco, A.P., and Kunkel, L.M. 1988. The complete sequence of dystrophin predicts a rod-shaped cytoskeletal protein. Cell. 53:219-228.

3. Koenig, M., et al. 1987. Complete cloning of the Duchenne muscular dystrophy (DMD) cDNA and preliminary genomic organization of the DMD gene in normal and affected individuals. Cell. 50:509-517.

4. Hoffman, E.P. 1991. Molecular diagnostics of Duchenne/Becker dystrophy: new additions to a rapidly expanding literature. J. Neurol. Sci. 101:129-132.

5. Prior, T.W., et al. 1995. Spectrum of small mutations in the dystrophin coding region. Am. J. Hum. Genet. 57:22-33.

6. Sicinski, P. et al. 1989. The molecular basis of muscular dystrophy in the $m d x$ mouse: a point mutation. Science. 244:1578-1580.

7. Karpati, G., Carpenter, S., and Prescott, S. 1988. Small-caliber skeletal fibers do not suffer necrosis in $m d x$ mouse dystrophy. Muscle Nerve. 11:795-803.

8. Petrof, B.J., Shrager, J.B., Stedman, H.H., Kelly, A.M., and Sweeney, H.L. 1993. Dystrophin protects the sarcolemma from stresses developed dur- ing muscle contraction. Proc. Natl. Acad. Sci. USA 90:3710-3714.

9. Stedman, H.H., et al. 1991. The $m d x$ mouse diaphragm reproduces the degenerative changes of Duchenne muscular dystrophy. Nature. 352:536-539.

10. Palmer, E., Wilhelm, J.M., and Sherman, F. 1979. Phenotypic suppression of nonsense mutants in yeast by aminoglycoside antibiotics. Nature. 277:148-150.

11. Singh, A., Ursic, D., and Davies, J. 1979. Phenotypic suppression and misreading in Saccharomyces cerevisiae. Nature. 277:146-148.

12. Bedwell, D.M., et al. 1997. Suppression of a CFTR premature stop mutation in a bronchial epithelial cell line. Nat. Med. 3:1280-1284.

13. Howard, M., Frizzell, R.A., and Bedwell, D.M. 1996. Aminoglycoside antibiotics restore CFTR function by overcoming premature stop codons. Nat. Med. 2:467-469.

14. Neville, C., Rosenthal, N., McGrew, M., Bogdanova, N., and Hauschka, S. 1998. Skeletal muscle cultures. In Methods in cell biology. Volume 52. H.L. Sweeney and C. Emerson, editors. Academic Press. San Diego, CA. 85-116.

15. Sweeney, H.L., and Feng, H. 1998. Structure-function analysis of cytoskeletal/contractile proteins in avian myotubes. In Methods in cell biology. Volume 52. H.L. Sweeney and C. Emerson, editors. Academic Press. San Diego, CA. 275-282.

16. Morris, T.H. 1995. Antibiotic therapeutics in laboratory animals. Lab. Anim. 29:16-36.

17. Swan, S.K. 1997. Aminoglycoside nephrotoxicity. Semin. Nephrol. 17:27-33.

18. Barton-Davis, E.R., Shoturma, D.I., Musaro, A., Rosenthal, N., and Sweeney, H.L. 1998. Viral-mediated expression of insulin-like growth factor I blocks the aging-related loss of skeletal muscle function. Proc. Natl. Acad. Sci. USA. 95:15603-15607.

19. Ervasti, J.M., and Campbell, K.P. 1991. Membrane organization of the dystrophin-glycoprotein complex. Cell. 66:1121-1131.

20. Hoffman, E.P., Brown, R.H., Jr., and Kunkel, L.M. 1987. Dystrophin: the protein product of the Duchenne muscular dystrophy locus. Cell. 51:919-928.

21. McNally, E.M., et al. 1996. Mutations that disrupt the carboxyl-terminus of $\gamma$-sarcoglycan cause muscular dystrophy. Hum. Mol. Genet. 5:1841-1847.

22. Rafael, J.A., et al. 1994. Prevention of dystrophic pathology in $m d x$ mice by a truncated dystrophin isoform. Hum. Mol. Genet. 3:1725-1733.

23. Song, B., and Schacht, J. 1996. Variable efficacy of radical scavengers and iron chelators to attenuate gentamicin ototoxicity in guinea pig in vivo. Hear. Res. 94:87-93.

24. Pearce, R.A., Finley, R.J., Mustard, R.A., and Duff, J.H. 1985. 2,3-dihydroxybenzoic acid. Arch. Surg. 120:937-940.

25. Hauser, M.A., and Chamberlain, J.S. 1996. Progress towards gene therapy for Duchenne muscular dystrophy. J. Endocrinol. 149:373-378.

26. Karpati, G., and Ascadi, G. 1993 . The potential for gene therapy in Duchenne muscular dystrophy and other genetic muscle diseases. Muscle Nerve. 16:1141-1153.

27. Hauser, M.A., Amalfitano, A., Kumar-Singh, R., Hauschka, S.D., and Chamberlain, J.S. 1997. Improved adenoviral vectors for gene therapy of Duchenne muscular dystrophy. Neuromuscul. Disord. 7:277-283.

28. Phelps, S.F., et al. 1995. Expression of full-length and truncated dystrophin mini-genes in transgenic mdx mice. Hum. Mol. Genet. 4:1251-1258.

29. Tuffery, S. et al. 1998. Mutation analysis of the dystrophin gene in Southern French DMD or BMD families: from Southern blot to protein truncation test. Hum. Genet. 102:334-342. 